\title{
Search for Heavy Stable Charged Particles in CMS
}

\author{
Norbert Neumeister*i \\ Department of Physics, Purdue University, W. Lafayette, IN, USA \\ E-mail: neumeistepurdue.edu
}

The result of a search at the LHC for heavy stable charged particles produced in pp collisions at $\sqrt{s}=7 \mathrm{TeV}$ is described. The data sample was collected with the CMS detector and corresponds to an integrated luminosity of $3.1 \mathrm{pb}^{-1}$. Momentum and ionization-energy-loss measurements in the inner tracker detector are used to identify tracks compatible with heavy slow-moving particles. Additionally, tracks passing muon identification requirements are also analyzed for the same signature. In each case, no candidate passes the selection, with an expected background of less than 0.1 events. A lower limit at the $95 \%$ confidence level on the mass of a stable gluino is set at $398 \mathrm{GeV} / \mathrm{c}^{2}$, using a conventional model of nuclear interactions that allows charged hadrons containing this particle to reach the muon detectors. A lower limit of $311 \mathrm{GeV} / \mathrm{c}^{2}$ is also set for a stable gluino in a conservative scenario of complete charge suppression, where any hadron containing this particle becomes neutral before reaching the muon detectors.

Workshop on Discovery Physics at the LHC - Kruger 2010

December 05-10, 2010

Kruger National Park, Mpumalanga, South Africa

\footnotetext{
*Speaker.

${ }^{\dagger}$ for the CMS collaboration
} 


\section{Introduction}

Heavy stable (or quasi-stable) charged particles (HSCPs) appear in various extensions of the standard model (SM) [1]. If the lifetime of an HSCP produced at the Large Hadron Collider (LHC) is longer than a few nanoseconds, the particle will travel over distances that are comparable or larger than the size of a typical particle detector. In addition, if the HSCP mass is $>100 \mathrm{GeV} / \mathrm{c}^{2}$, a significant fraction of these particles will have a velocity, $\beta \equiv v / c$ smaller than 0.9 . These HSCPs will be directly observable through the distinctive signature of a high momentum particle with an anomalously large rate of energy loss through ionization $(d E / d x)$.

HSCPs with strong charge will hadronize and form mesons, baryons or gluonballs. The hadronized states are generically called $R$-hadrons. Since the particle loses energy primarily through low momentum-transfer interactions, even if strongly interacting, it will be highly penetrating and will likely be identified as a muon. The interactions with matter experienced by a stronglyinteracting $R$-hadron can lead to it flipping the sign of its electric charge or becoming neutral. A recent study [2] on the modeling of nuclear interactions of HSCPs traveling through matter, favours a scenario of charge suppression. In this model the majority of $R$-hadrons containing a gluino, or a supersymmetric bottom squark, are expected to emerge as neutral particles after traversing the detector. If this model is correct, the majority of these HSCPs would not be observed in the muon system of a typical collider detector. Experimental strategies that do not rely on the muon-like behavior for the HSCPs are therefore of great importance.

We present a search for HSCPs produced in pp collisions at $\sqrt{s}=7 \mathrm{TeV}$ at the LHC with the Compact Muon Solenoid (CMS) detector. A detailed description of the CMS detector can be found in [3]. The search is based on the data sample collected between April and August 2010 corresponding to an integrated luminosity of $3.1 \mathrm{pb}^{-1}$. The analysis makes use of two approaches. In a first selection, referred to as tracker-only, the HSCP candidates are searched for as individual tracks reconstructed in the inner tracker detector with large $d E / d x$ and $p_{\mathrm{T}}$. A second selection, referred to as tracker-plus-muon, additionally requires that the track is identified as a muon in the outer muon detector. For both selections, the mass of the candidate is calculated from the measured $p$ and $d E / d x$.

\section{Analysis}

The events used in this analysis are collected with triggers requiring: a high- $p_{\mathrm{T}}$ muon $\left(p_{\mathrm{T}}>\right.$ $9 \mathrm{GeV} / c$ ); or a dimuon pair ( $p_{\mathrm{T}}>3 \mathrm{GeV} / c$ for each muon); or calorimeter-based missing transverse energy $\left(E_{\mathrm{T}}^{\text {miss }}>100 \mathrm{GeV}\right)$, to search for HSCPs failing muon identification or emerging mainly as neutral particles after traversing the calorimeters; or a high-transverse-energy jet $\left(E_{\mathrm{T}}>100 \mathrm{GeV}\right)$ to search for HSCPs accompanied by substantial hadronic activity.

Candidate HSCPs are pre-selected by requiring a track with $|\eta|<2.5, p_{\mathrm{T}}>15 \mathrm{GeV} / c$, relative uncertainty on the $p_{\mathrm{T}}$ less than $15 \%$, and transverse (longitudinal) impact parameter with respect to the reconstructed primary collision vertex less than $0.25(2.0) \mathrm{cm}$. Candidate tracks are also required to have at least three measurements in the silicon-strip detector. For the tracker-plus-muon selection, we additionally require the track to be compatible with track segments reconstructed in the muon system. As an estimator of the degree of compatibility of the observed charge measure- 
ments with the MIP hypothesis, a modified version of the Smirnov-Cramer-von Mises [4] discriminant is used:

$$
I_{a s}=\frac{3}{N} \times\left(\frac{1}{12 N}+\sum_{i=1}^{N}\left[P_{i} \times\left(P_{i}-\frac{2 i-1}{2 N}\right)^{2}\right]\right),
$$

where $N$ is the number of charge measurements in the silicon-strip detectors, $P_{i}$ is the probability for a MIP to produce a charge smaller or equal to the $i$-th charge measurement for the observed path length in the detector, and the sum is over the track measurements ordered in terms of increasing $P_{i}$. Non-relativistic HSCP candidates will have the value of the discriminant $I_{a s}$ approaching unity. The charge probability density function used to calculate $P_{i}$ is obtained using tracks with $p>5 \mathrm{GeV} / c$ in events collected with a minimum bias trigger. Figure 1 shows normalized distributions of $p_{\mathrm{T}}$ and $I_{a s}$ in data and two MC samples, for candidates passing the tracker-only pre-selection. The first MC sample contains events from QCD processes. The second MC sample contains signal events from pair-production of stable gluinos with a mass of $200 \mathrm{GeV} / \mathrm{c}^{2}$. The MC QCD simulations are found to reproduce the data, and the simulated signal is clearly separated.
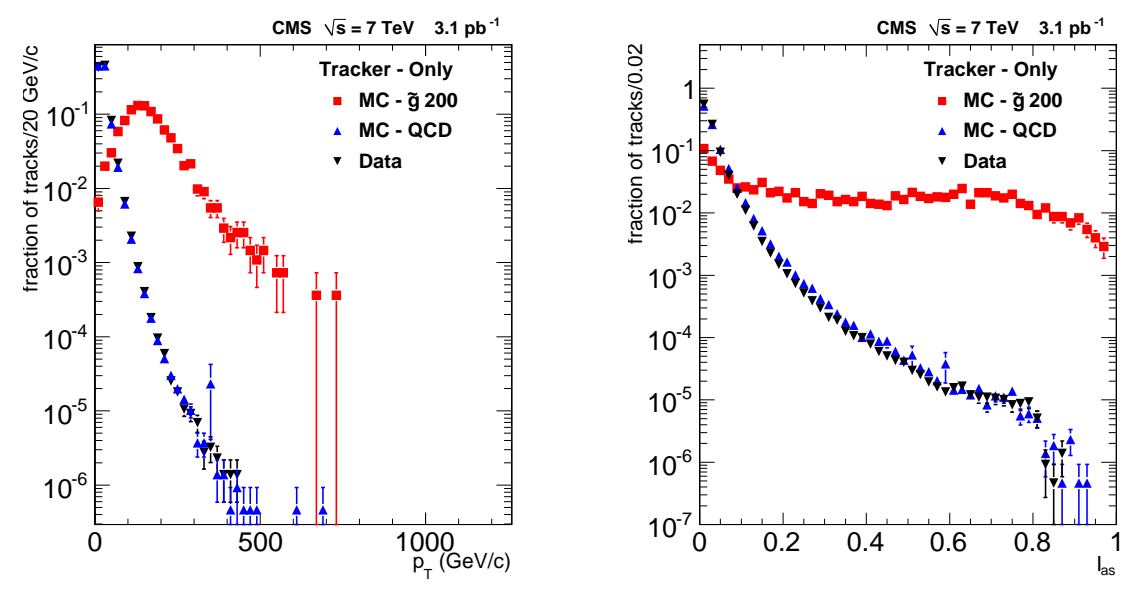

Figure 1: Normalized distributions of $p_{\mathrm{T}}$ (left) and $I_{a s}$ (right) in data and two MC samples, for candidates passing the tracker-only pre-selection. The two MC samples contain events from QCD processes and from gluino pair-production with a mass of $200 \mathrm{GeV} / \mathrm{c}^{2}$, respectively.

The most probable value of the particle $d E / d x$ is determined using a harmonic estimator $I_{h}$ of grade $k=-2$ :

$$
I_{h}=\left(\frac{1}{N} \sum_{i} c_{i}^{k}\right)^{1 / k}
$$

where $c_{i}$ is the charge per unit path length in the detector of the $i$-th measurement for a given track. In order to estimate the mass $(m)$ of highly ionizing particles, the following relationship between $I_{h}, p$, and $m$ is assumed:

$$
I_{h}=K \frac{m^{2}}{p^{2}}+C
$$

Equation 2.3 reproduces the Bethe-Bloch formula with an accuracy of better than $1 \%$ in the range $0.4<\beta<0.9$, which corresponds to $1.1<(d E / d x) /(d E / d x)_{\mathrm{MIP}}<4.0$. The empirical parameters $K$ and $C$ are determined from data using a sample of low-momentum protons, for which the fitted 
values are $K=2.579 \pm 0.001 \mathrm{MeV} \mathrm{cm}^{-1} c^{2}$ and $C=2.557 \pm 0.001 \mathrm{MeV} \mathrm{cm}^{-1}$, and the mass resolution is $7 \%$. For masses above $100 \mathrm{GeV} / c^{2}$, the mass resolution is expected to worsen because of the deterioration of the momentum resolution and because of the limit on the maximum charge that can be measured by the silicon strip tracker ADCs, which also affects the mass scale. These effects are taken into account by the MC: for a $300 \mathrm{GeV} / \mathrm{c}^{2} \mathrm{HSCP}$, the mass resolution and the reconstructed peak position are found to be $12 \%$ and $265 \mathrm{GeV} / c^{2}$, respectively.

The search is performed as a counting experiment. Signal candidates are required to have $I_{a s}$ and $p_{\mathrm{T}}$ greater than threshold values and the mass to be in the range of 75 to $2000 \mathrm{GeV} / c^{2}$, allowing sensitivity to HSCP masses as low as $100 \mathrm{GeV} / c^{2}$. The $I_{a s}$ distribution for the pre-selected tracks, and in particular its tail, depends strongly on the number of charge measurements on the track. Thus, to increase the sensitivity of the search, pre-selected tracks are divided into subsamples according to the number of silicon strip measurements. The $I_{a s}\left(p_{\mathrm{T}}\right)$ threshold in each subsample is determined by requiring a constant efficiency on data for all subsamples, when the threshold is applied separately. A method that exploits the absence of correlation between the $p_{\mathrm{T}}$ and $d E / d x$ measurements in data is used to estimate the background from MIPs. In a given subsample $j$, the number of tracks that are expected to pass both the final $p_{\mathrm{T}}$ and $I_{a s}$ thresholds set for the subsample is estimated as $D_{j}=B_{j} C_{j} / A_{j}$, where $A_{j}$ is the number of tracks that fail both the $I_{a s}$ and $p_{\mathrm{T}}$ selections and $B_{j}\left(C_{j}\right)$ is the number of tracks that pass only the $I_{a s}\left(p_{\mathrm{T}}\right)$ selection. The $B_{j}$ and $C_{j}$ tracks are then used to form a binned probability density function in $I_{h}(p)$ for the $D_{j}$ tracks. Finally, using the mass determination (Eq. 2.3), the full mass spectrum of the background in the signal region $D$ is predicted. By comparing the predicted and observed number of tracks for several very loose selections in a control region of the mass spectrum, corresponding to masses below $75 \mathrm{GeV} / \mathrm{c}^{2}$, the prediction is found to underestimate systematically the observation by $12 \%$ $(5 \%)$ for the tracker-only (tracker-plus-muon) selection. After correcting the predicted background by this amount, the remaining background systematic uncertainty is conservatively estimated as twice the r.m.s. of the prediction-to-observation ratio distribution The resulting uncertainty on the predicted background is $14 \%(17 \%)$.

As significant background rejection can be obtained without a sizable effect on the signal efficiency, the final selection is optimized by requiring the total expected background in the search region to be $\sim 0.05$ events. This low-background choice optimizes the discovery potential even if just a handful of events are observed, and at the same time maintains significant exclusion sensitivity in the case that no events are observed.

\section{Results}

In addition to the final tight selection, a loose selection, retaining a relatively large number of background candidates, was applied which allows us to compare the background prediction with the observed data. Figure 2 shows good agreement between the observed and predicted mass spectrum obtained using the loose selection for the tracker-plus-muon and tracker-only candidates.

No candidate HSCP track is observed in either the tracker-only or tracker-plus-muon analysis. Given the null result, cross section upper limits at the 95\% C.L. are set on the HSCP production for two benchmark scenarios: direct production of $\tilde{\mathrm{g}}$ pairs and supersymmetric top squark $\left(\mathfrak{t}_{1}\right)$ 

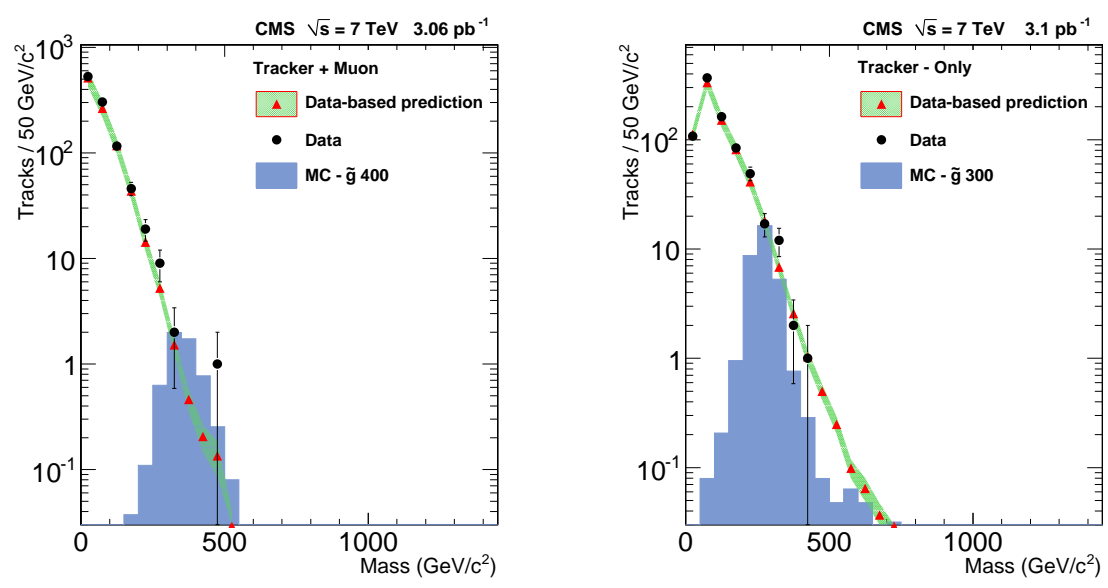

Figure 2: Mass spectrum for the loose selection for the tracker-plus-muon (left) and tracker-only (right) candidates. Shown are: observed spectrum (black dots with the error bars), data-based predicted background spectrum (red triangles) with its uncertainty (green band) and the spectrum predicted by MC for a signal of pair-produced stable $\tilde{g}$ with a mass of 400 (left) and 300 (right) $\mathrm{GeV} / \mathrm{c}^{2}$ (blue histogram).

pairs. The upper limit on the cross section is computed using a Bayesian method with a flat signal prior and a log-normal prior used for integration over the nuisance parameters [4]. In order to obtain a conservative upper limit the expected background was set to zero. The tracker-plus-muon selection provides better limits than the tracker-only for all scenarios but the one with complete charge suppression. For each considered scenario, the cross section upper limit obtained with the most sensitive selection is reported in Fig. 3, along with the theoretical predictions for $\tilde{\mathrm{g}}$ and $\tilde{\mathrm{t}}_{1}$ pair production computed using the PROSPINO program [5]. The $\tilde{g}$ theoretical predictions refer to the case where the squarks and gluino are degenerate in mass. In the heavy squark limit these cross sections are about $10 \%$ higher. For the case of $\tilde{\mathfrak{t}}_{1}$, beyond LO, the cross section does not only depend on the $\tilde{\mathrm{t}}_{1}$ mass, but also, though to a much lesser extent, on the $\tilde{\mathrm{g}}$ mass, the average mass of the first and second generation squarks and the stop mixing angle. For this reason, the $\tilde{\mathrm{t}}_{1}$ theoretical predictions reported in Fig. 3 refer to the SPS1a' benchmark scenario [6].

The main source of systematic uncertainties are: 15\% signal acceptance (including trigger, momentum scale and reconstruction efficiency), $11 \%$ absolute value of the integrated luminosity, and 10-25\% theoretical cross section. All systematic uncertainties are included in the cross section upper limits reported in Fig. 3. From the intersection of the cross section limit curve and the lower edge of the theoretical cross section band we set a 95\% C.L. lower limit of 398 (357) GeV/c $c^{2}$ on the mass of pair-produced $\tilde{\mathrm{g}}$ with $f=0.1(0.5)$, using the tracker-plus-muon selection. The analogous limit on the $\tilde{\mathrm{t}}_{1}$ mass is $202 \mathrm{GeV} / \mathrm{c}^{2}$.

\section{Conclusions}

In summary, the CMS detector has been used to identify highly ionizing, high- $p_{\mathrm{T}}$ particles and measure their masses. Two searches have been conducted: a very inclusive and

model independent one that uses highly-ionizing tracks reconstructed in the inner tracker detector, and another requiring also that these tracks be identified in the CMS muon system. 


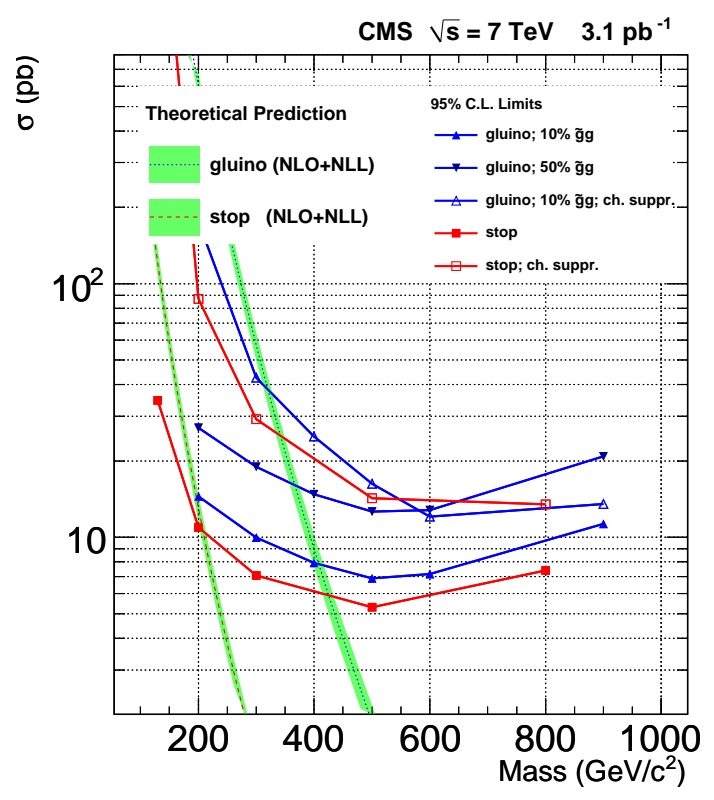

Figure 3: Predicted theoretical cross section and observed 95\% C.L. upper limits on the cross section for the different combinations of models and scenarios considered: pair production of supersymmetric stops and gluinos; different fractions, $f$, of $R$-gluonball states produced after hadronization and charge suppression scenarios. Only the results obtained with the most sensitive selection are reported: tracker-only for the charge suppression scenarios and tracker-plus-muon for all other cases. The bands represent the theoretical uncertainties on the cross section values.

In each case, the observed distribution of the candidate masses is consistent with the expected background. We have set lower limits on masses of stable strongly interacting supersymmetric particles. For the case of $\tilde{\mathrm{g}}$ with $f=0.1$ and $\tilde{\mathfrak{t}}_{1}$, a lower mass limit of 398 and $202 \mathrm{GeV} / \mathrm{c}^{2}$, respectively, is set at the $95 \%$ C.L. with the analysis that uses muon identification. In a pessimistic scenario of complete charge suppression the above $\tilde{\mathrm{g}}$ mass limit is reduced to $311 \mathrm{GeV} / \mathrm{c}^{2}$ and is obtained with the tracker-only selection.

\section{References}

[1] M. Fairbairn et al., "Stable massive particles at colliders", Phys. Rept. 438 (2007) 1-63.

[2] R. Mackeprang and D. Milstead, "An Updated Description of Heavy-Hadron Interactions", Eur. Phys. J. C66 (2010) 493.

[3] CMS Collaboration, "The CMS experiment at the CERN LHC”, JINST 0803 (2008) S08004.

[4] F. James, "Statistical Methods in Experimental Physics", World Scientific, Singapore, 178 (2006).

[5] W. Beenakker, R. Hopker, and M. Spira, "PROSPINO: A program for the PROduction of Supersymmetric Particles in Next-to-leading Order QCD”, arXiv:hep-ph/9611232 (1996).

[6] J.A. Aguilar-Saavedra et al., "Supersymmetry parameter analysis: SPA convention and project", Eur. Phys. J. C46 (2006) 43. 